\title{
Guest editorial: mobile computing support for geospatial systems
}

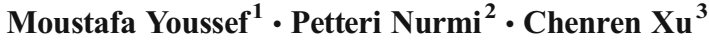

Published online: 27 February 2017

(C) Springer Science+Business Media New York 2017

The integration of wireless communications into mobile devices has opened the door for a revolution of geospatial systems. Specifically, contemporary mobile devices come equipped with a large array of sensors, are connected everywhere, and are carried around by their users almost 24/7. Taken together, these developments enable a number of new paradigms such as crowd-sensing, anywhere (or ubiquitous) context-awareness, and large scale machine-tomachine interaction. These paradigms in turn pose novel challenges and opportunities for geospatial systems.

This special issue includes four papers covering both theoretical, experimental and visionary research in the area of mobile computing support for geospatial systems.

Searching for spatially located resources is a common yet frustrating everyday chore for many people. Consider, e.g., finding a parking place in a crowded city, a taxi driver looking for the next passenger, or an electric car owner trying to find a charging station. What further makes the task in these examples challenging is that the availability of the resources varies dynamically and obtaining accurate information about them is not feasible, e.g., due to deployment cost of sensors, parallel searchers by multiple users, and the rate at which changes in availability occur. The authors of "Probabilistic Spatio-Temporal Resource Search" (DOI 10.1007/S10707-016-0275-9) address the provisioning of decision support

\section{Moustafa Youssef}

moustafa.youssef@gmail.com

Petteri Nurmi

petteri.nurmi@cs.helsinki.fi

Chenren $\mathrm{Xu}$

chenren@pku.edu.cn

1 Egypt-Japan University of Science and Technology (E-JUST), New Borg Elarab City 21934 Alexandria, Egypt

2 University of Helsinki, Helsinki, Finland

3 Peking University, Beijing, China 
for this kind of search tasks, providing efficient algorithms for finding optimal search paths that maximize the probability of finding resources while at the same minimize the cost of searching. Experiments are carried out using a combination of parking availability data and GPS traces of cabs. The experiments demonstrate that the proposed algorithms significantly reduce search times compared to a baseline technique and improve robustness at higher levels of resource unavailability.

Over the past decade, with rapid development of sensor built-in mobile devices, wireless adhoc sensor networks and open sensor platforms; the traditional technocratic Geosensor webs now are experiencing a transition towards an emerging end-user-oriented Geosensor media, through which ordinary users are able to freely process, recreate and disseminate sensor data and information for self-expression, collaborative tasks or collective wisdom, etc. In the paper "A Semantic HTML based Approach for Geosensor Media” (DOI 10.1007/S10707-016-0273-Y), the authors discuss the concept, implication and potential issues of Geosensor media. A general framework involving semantic interaction, geospatial interface and participatory sensing is brought out, and a typical prototype is implemented and evaluated.

When large-scale disasters occur, evacuees have to evacuate to safe places quickly. They, however, may not be able to afford to obtain sufficient information for their evacuations under such emergent situations. The paper "Automatic Evacuation Guiding Scheme Based On Implicit Interactions Between Evacuees and Their Mobile Nodes" (DOI 10.1007/S10707-016-0270-1) proposes an automatic evacuation guiding scheme using evacuees' mobile nodes, e.g., smart phones. The key idea to achieve automatic evacuation guiding is implicit interactions between evacuees and their mobile nodes. Each mobile node tries to navigate its evacuee by presenting an evacuation route. At the same time, it can also trace the actual evacuation route of the evacuee by measuring his/her positions periodically. The proposed scheme automatically estimates blocked road segments from the difference between the presented evacuation route and the actual evacuation route, and then recalculates the alternative evacuation route. In addition, evacuees also share such information among themselves through direct wireless communication with other mobile nodes and that with a server via remaining communication infrastructures.

Due to the enormous penetration of connected computing devices with diverse sensing and localization capabilities, a good fraction of an individual's activities, locations, and social connections can be sensed and spatially pinpointed. The paper "Towards Pervasive Geospatial Affect Perception" (DOI 10.1007/s10707-017-0294-1) investigates the detection of emotion and sentiment from environmental, on-body and smartphone sensors and proposes an emotional map as an interface accumulating and interpreting the enormous amount of emotionrelated data from diverse distributed and mobile sensing sources. The paper first surveys existing work on emotional sensing and geospatial systems. Then it presents a taxonomy of large-scale emotional sensing. The paper also discusses model relationships among human emotions and geo-spaces using networks, apply clustering algorithms to the networks and visualize clusters on a map considering space, time and mobility. Two studies exploiting environmental and on-body sensors are used to evaluate the proposed emotion recognition framework.

We hope that this special issue will be appealing to both experts and application developers of mobile computing support for geospatial systems. 
Moustafa Youssef is the Founder \& Director of the Wireless Research Center at Egypt-Japan University of Science and Technology (E-JUST). His research interests include mobile computing, location determination technologies, pervasive computing, mobile wireless networks, and network security. He is an associate editor for the ACM TSAS, a previous area editor of the ACM MC2R and served on the organizing and technical committees of numerous prestigious conferences. Prof. Youssef is the recipient of the 2003 University of Maryland Invention of the Year award, the 2010 TWAS-AAS-Microsoft Award for Young Scientists, the 2012 Egyptian State Award, the 2013 and 2014 COMESA Innovation Awards, the 2013 ACM SIGSpatial GIS Conference Best Paper Award, among many others. He is also an ACM Distinguished Speaker and an ACM Distinguished Scientist.

Petteri Nurmi is a Senior Researcher in the Department of Computer Science at the University of Helsinki, Finland where he leads the Ubiquitous Sensing Research Group. His research interests include mobile sensing, adaptive interfaces, and ubiquitous computing. Dr. Nurmi regularly serves on organizing and technical committees of several prestigious conferences. He is a recipient of the Mark Weiser best paper award at IEEE PerCom in 2015. He holds a Ph.D. degree in Computer Science from the University of Helsinki in 2009.

Chenren Xu is an Assistant Professor in the School of Electronics Engineering and Computer Science and also a member of Center for Energy-efficient Computing and Applications (CECA) at Peking University. His current research Interests are in Internet-of-Things architecture, mobile cloud computing and connected health. He earned a Ph.D. in Electrical and Computer Engineering and a M.S. in Applied Mathematical Statistics from Rutgers University in 2014, and a B.E. in Automation from Shanghai University in 2008. He was a Postdoctoral Research Fellow in the School of Computer Science at Carnegie Mellon University from 2014 to 2015. 